\title{
Relations entre le stade de développement du liseron des champs (Convolvulus arvensis $L$.) et le comportement du 2,4-D et du glyphosate dans les plantes
}

\author{
L. M. Tamayo Esquer ${ }^{1}$ et P. Gaillardon² \\ 1 CIANO, calle Norman E. Borlaug, Km 12 AP 515, Cd. Obregon, Sonora, Mexique \\ 2 Laboratoire des Herbicides, INRA, BV 1540, 21034 Dijon Cedex, France
}

(reçu le 10-8-1988, accepté le 4-10-1988)

Résumé - L'absorption, le transport et le métabolisme du 2,4-D et du glyphosate dans le liseron des champs (Convolvulus arvensis) ont été étudiés en déposant les herbicides radioactifs sur des feuilles portées par les tiges jeunes ou âgées de deux cycles successifs de repousse. Ces phénomènes sont relativement rapides et durent souvent moins de trois jours. Le transport des herbicides est principalement basipète et le glyphosate atteint mieux les organes souterrains des plantes que le 2,4-D. Un transport acropète significatif se produit dans les jeunes tiges et dépend, dans le cas du 2,4-D, du cycle de repousse. A ce stade, le 2,4-D s'accumule dans les dix premiers centimètres des organes souterrains. Le transport des herbicides dans les tiges âgées est presque exclusivement basipète, sauf lorsque le 2,4-D est déposé sur des feuilles proches de l'apex. A ce stade, cet herbicide pénètre mal dans les feuilles. II atteint d'autant mieux les organes souterrains du liseron que les feuilles traitées en sont plus proches et il se distribue mieux dans ces organes qui jouent probablement un rôle déterminant dans le transport des deux herbicides. La dégradation du 2,4-D dans les plantes ne met pas en jeu l'hydroxylation des molécules mais peut limiter le transport de cet herbicide. Au contraire, la stabilité du glyphosate peut favoriser la mobilité de ce dernier.

\section{2, 4-D - glyphosate - Convolvulus arvensis L. - transport - métabolisme}

\begin{abstract}
Summary - Relationship between plant growth stage and 2,4-D and glyphosate behaviour in field bindweed (Convolvulus arvensis L.). Absorption, translocation and metabolism of 2.4-D and glyphosate in field bindweed (Convolvulus arvensis) have been studied when applying labelled herbicides to young or aged stems of two consecutive regrowth cycles. Phenomena occurred quickly (less than 3 days). Herbicide transport was mainly basipetal and glyphosate translocated into the roots better than 2.4-D. A significant acropetal transport occurred in young stems and with 2.4-D, this depended on growth cycle. At this stage, 2.4-D accumulated in the 0-10 cm root fraction. In aged stems, transport of both herbicides was almost entirely basipetal except when 2.4-D was applied to leaves near the stem tip. At this stage, absorption of 2.4-D decreased, translocation into roots increased when leaf-root length decreased and herbicide showed a better distribution in roots. The root system of field bindweed probably plays a basic role in translocation of both herbicides. Breakdown of 2.4-D in plants did not involve hydroxylation but might prevent herbicide translocation. Glyphosate stability, on the other hand, is likely to favour transport.
\end{abstract}

2.4-D - glyphosate - Convolvulus arvensis L. - translocation - metabolism

\section{Introduction}

Le liseron des champs (Convolvulus arvensis) est une mauvaise herbe pérenne largement répandue dans les cultures. II possède des organes souterrains très développés, persistants et capables de produire de nombreuses repousses, ce qui rend son contrôle particulièrement difficile. La lutte chimique contre cette espèce requiert des herbicides mobiles dans le phloème, capables d'atteindre les organes souterrains des plantes et d'agir sur ces organes. 
Comme dans le cas des annuelles, l'action des herbicides sur les mauvaises herbes pérennes dépend du stade de développement des plantes mais alors que les premières sont généralement plus sensibles lorsqu'elles sont jeunes, les secondes sont souvent mieux détruites au début de la floraison (Aberg et Stecko, 1976).

L'absorption, le transport et le métabolisme des herbicides dans les plantes jouent un rôle important dans l'action phytotoxique. De ce fait, la diminution de l'absorption foliaire au cours du développement des feuilles (Baker et Hunt, 1981; Duncan et al., 1981) peut expliquer au moins partiellement la moindre sensibilité des mauvaises herbes annuelles âgées. Chez les plantes pérennes, le transport est particulièrement déterminant et peut changer selon le stade de développement des plantes. Ce phénomène a été observé avec le 2,4-D chez Convolvulus arvensis L. (Muller et Guncan, 1973), avec le clopyralide chez Cirsium arvense $L$. (O' Sullivan et Kossatz, 1984) et avec le glyphosate chez Cirsium arvense L. (Sprankle et al., 1975; McAllister et Haderlie, 1985) et Sorghum halepense L. (Lolas et Coble, 1980). II est susceptible d'influencer l'efficacité des herbicides.

Cette étude se propose d'observer les modifications du comportement, c'est-à-dire de l'absorption foliaire, du transport et du métabolisme, de deux herbicides systémiques : le 2,4-D et le glyphosate, dans le liseron des champs (Convolvulus arvensis L.), en fonction du stade de développement des plantes concernant à la fois les organes aériens et souterrains.

\section{Matériels et Méthodes}

\section{Culture des plantes}

Les graines de liseron ont été récoltées dans l'Etat de Sonora (Mexique). Elles ont été trempées dans l'acide sulfurique concentré pendant $20 \mathrm{~min}$, puis soigneusement rincées à l'eau et mises à germer dans des boîtes de Pétri, en serre chauffée. Chaque graine germée a été repiquée dans un pot en matière plastique $(20 \times 20 \times 18 \mathrm{~cm})$ rempli d'un mélange sable / terre argilolimoneuse (2/1). Les plantes, cultivées en serre, ont été arrosée régulièrement avec de l'eau et, une fois par semaine, avec la solution nutritive de Hoagland (Agbakoba et Goodin, 1969).

Trois mois après le repiquage, l'unique tige fleurie de chaque plante a été rabattue pour permettre une première repousse comprenant deux types de tiges : les unes issues de la base de la tige précédemment coupée et les autres constituées par des drageons (Fig. 1).
Après trois mois de croissance, les tiges en fleurs ont été de nouveau rabattues pour permettre une deuxième repousse comprenant trois types de tiges : les unes issues des tiges coupées, les autres issues des drageons coupés et les dernières constituées par de nouveaux drageons (Fig. 1).

Influence du stade de développement des plantes sur le comportement des herbicides

Le comportement du 2,4-D et du glyphosate a été étudié dans les premières et deuxièmes repousses de liseron, dont la masse des organes souterrains était nettement différente, en appliquant les herbicides sur des tiges jeunes (environ $50 \mathrm{~cm}$ ) ou âgées (environ $150 \mathrm{~cm}$, stade début floraison).

A chaque stade, les plantes ont été traitées au 2,4-D (2 I ha-1 d'un sel de diméthylamine formulé : $U 46 \mathrm{D}$, à $480 \mathrm{~g} \mathrm{~F}^{-1}$ de m.a.) ou au glyphosate (10 I ha-1 d'un sel d'isopropylamine : Roundup, à $360 \mathrm{~g} \mathrm{H}^{-1}$ de m.a.), par pulvérisation ( $400 \mathrm{I} \mathrm{ha} \mathrm{h}^{-1}$ d'eau), à l'exception des feuilles destinées à recevoir les herbicides radioactifs, qui étaient protégées par un film plastique. Immédiatement après le traitement, les plantes ont été placées dans une chambre climatisée à $20^{\circ} \mathrm{C}, 80 \%$ d'humidité relative et $16 \mathrm{~h}$ de lumière (15 000 lux). Les herbicides radioactifs ont été appliqués sur deux ou trois tiges des plantes issues des premières ou secondes repousses, correspondant respectivement aux divers types de tige formés par ces repousses. Pour chaque plante, une solution
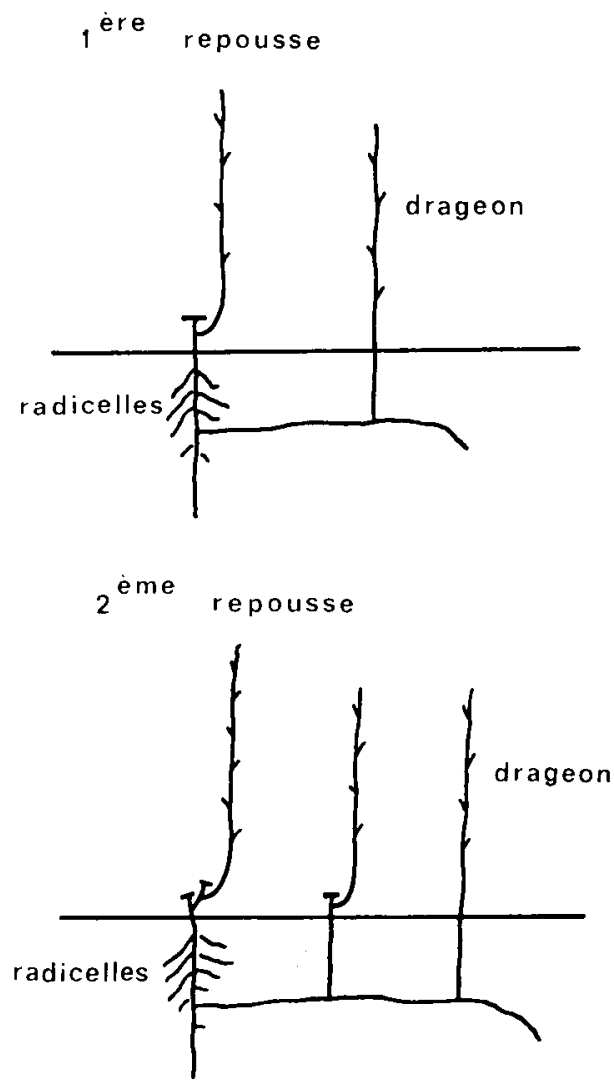

Fig. 1. Représentation schématique des premières et deuxièmes repousses de liseron. 
contenant le 2,4-D ou le glyphosate marqués, a été déposée à l'aide d'une séringue Hamilton sur quatre feuilles consécutives $(5 \mu$ répartis en petites gouttelettes sur chaque feuille) situées à mi-hauteur de chaque tige traitée.

La solution radioactive de 2,4-D a été obtenue en mélangeant des solutions équimoléculaires d'herbicide marqué au carbone 14 sur le cycle (Amersham, 777 $\mathrm{MBq} \mathrm{mM}^{-1}, 97 \%$ de pureté radiochimique) et de diméthylamine, puis en ajoutant une solution de $U 46 \mathrm{D}$ pour atteindre une concentration finale de $2,4 \mathrm{~g} \mathrm{l}^{-1}$ de m.a. avec $8,88 \mathrm{MBq} \mathrm{ml}-1$ (traitement des tiges jeunes) ou $9,62 \mathrm{MBq} \mathrm{ml}-1$ (traitement des tiges âgées). Dans ce dernier cas, la préparation d'une solution de radioactivité volumique plus élevée avait pour but de limiter la dilution de la radioactivité dans les plantes, liée au plus grand développement des tiges et préjudiciable aux mesures. La solution de glyphosate a été obtenue de la même manière à partir de phosphonométhyle- ${ }^{-14} \mathrm{C}$-glyphosate fourni par la société Monsato $\left(72,9 \mathrm{MBq} \mathrm{mM}^{-1}\right.$, $94 \%$ de pureté radiochimique) et d'isopropylamine, puis en ajoutant une solution de Roundup pour atteindre la concentration finale de $9 \mathrm{~g} \mathrm{I}^{-1}$ de m.a. avec $0,43 \mathrm{MBq}$ $\mathrm{ml}^{-1}$ (traitement des tiges jeunes) ou $0,7 \mathrm{MBq} \mathrm{ml}-1$ (traitement des tiges âgées).

L'absorption foliaire, le transport et le métabolisme des herbicides radioactifs ont été déterminés dans chaque plante, 3 et 11 jours après le traitement. En raison des difficultés pratiques que présente ce type d'étude lorsque les plantes ont de grandes dimensions, les mesures de l'absorption et du transport n'ont été répétées que sur deux plantes, et le métabolisme n'a été analysé que dans une seule d'entre elles.

L'absorption du 2,4-D a été évaluée en lavant chaque feuille traitée au ${ }^{14} \mathrm{C}$ avec $10 \mathrm{ml}$ d'éthanol $80 \%$, pour déterminer la quantité de radioactivité restant à la surface des feuilles. Les liquides de lavage des 4 feuilles traitées d'une même tige ont été rassemblés et une fraction aliquote a été comptée par scintillation liquide dans le Dynagel (Baker Chemical). Pour le glyphosa te, les feuilles traitées d'une même tige ont été trempées dans $50 \mathrm{ml}$ d'eau pendant 2 min et la radioactivité dissoute a été mesurée par comptage d'un échantillon de la solution.

Le transport a été étudié en découpant chaque tige traitée au ${ }^{14} \mathrm{C}$ en 4 fractions : les feuilles traitées (FT), la fraction de la tige portant les feuilles traitées $(T)$, la tige et les feuilles situées plus haut (tige supérieure) ou plus bas (tige inférieure) que la fraction précédente. Les tiges non traitées avec les herbicides radioactifs (TNT) ont été rassemblées. Les organes souterrains de chaque plante ont été divisés en 4 fractions définies par leur éloignement de la base des tiges recevant les herbicides radioactifs : $0-10 \mathrm{~cm}, 10-20 \mathrm{~cm}, 20-40$ $\mathrm{cm}$, au-delà de $40 \mathrm{~cm}$, plus l'ensemble volumineux des radicelles $(R)$ issues de la partie supérieure de la racine principale formée par la jeune plantule. A chaque stade, les fractions ont été récoltées, pesées et conservées au congélateur. Celles de la première plante ont été lyophilisées et broyées puis des échantillons ont été brûlés au moyen d'un oxydizer afin de déterminer les quantités de radioactivité présentes. Les fractions de la deuxième plante ont été extraites par broyages successifs dans l'éthanol puis l'éthanol $80 \%(2,4-D)$ ou dans l'eau (glyphosate) jusqu'à épuisement de la radioactivité. Les extraits de chaque fraction, isolés après centrifugation, ont été rassemblés et une partie aliquote a été comptée. La radioactivité «insoluble" a été mesurée après combustion des culots de centrifugation séchés.

Le métabolisme des herbicides a été étudié dans les feuilles traitées, les autres parties des tiges et les organes souterrains de chaque plante soumise à l'extraction. Pour ce faire, les extraits correspondants ont été mélangés. L'analyse des métabolites du 2,4-D a été conduite selon une procédure décrite antérieurement (Hamilton et al., 1971); Leguay et Tyburn, 1981; Tamayo Esquer, 1986). Les extraits ont été évaporés à $\mathrm{sec}$, dissous dans $12 \mathrm{ml}$ d'une solution d'acide phosphorique à $\mathrm{pH} 2,5$ puis soumis à un partage avec $4 \times 20 \mathrm{ml}$ d'éther. Les composés radioactifs contenus dans les phases organiques et aqueuses ont été quantifiés par comptage de fractions aliquotes, puis concentrés dans un petit volume d'éthanol et d'éthanol $80 \%$, respectivement, avant d'être chromatographiés sur couche mince $(250 \mu)$ de gel de silice avec le mélange éluant éther/éthe de pétrole/acide formique (70/30/2) qui sépare les composés comme suit : $2,4-\mathrm{D}\left(R_{f}=0,54\right), 4 \mathrm{OH}$ 2,5-D $\left(R_{f}=0,53\right), 4 \mathrm{OH}-2,3-\mathrm{D}\left(R_{f}=0,35\right)$, conjugués aspartate et glutamate $\left(R_{f}=0,12\right.$ et 0,10$)$. L'identification du 2,4-D a été confirmée par chromatographie dans une deuxième dimension avec le mélange éluant chloroforme/méthanol/ammoniaque (75/25/2) qui sépare le $4 \mathrm{OH}-2,5-\mathrm{D}\left(R_{f}=0,06\right)$ du 2,4-D $\left(R_{f}=0,19\right)$. Les zones radioactives ont été repérées par autoradiographie et la silice a été grattée pour permettre le comptage de la radioactivité dans le Dynagel, après addition de $1 \mathrm{ml}$ d'éthanol $80 \%$. Dans quelques cas, les composés des phases organiques et aqueuses ont été hydrolysés dans $\mathrm{HCl} 6 \mathrm{~N}$ et $2 \mathrm{~N}$, respectivement pendant $24 \mathrm{~h}$ à $65^{\circ} \mathrm{C}$. Les hydrolysats ont été extraits à l'éther et les composés radioactifs ainsi récupérés ont été quantifiés et chromatographiés comme précédemment. Les extraits contenant le glyphosate ont été purifiés par addition de $50 \mathrm{mg} / \mathrm{ml}$ de résine Dowex $50 \mathrm{WX} 8 \mathrm{H}$ (Coupland, 1984) suivie d'une centrifugation. Les surnageants ont été concentrés puis chromatographiés sur couche mince de cellulose (Merck 15275) avec le mélange éluant éthanol/eau/ammoniaque $15 \mathrm{~N} / \mathrm{TCA}$ $(55 / 35 / 2,5 / 3,5)$ (Sprankle et al., 1978) qui sépare les composés comme suit : sarcosine $\left(R_{f}=0,59\right)$, glycine $\left(R_{f}=0,49\right)$, acide aminoéthylphosphonique $\left(R_{f}=0,36\right)$ et glyphosate $\left(R_{f}=0,24\right)$. Les zones radioactives ont été repérées, grattées et comptées comme précédemment après addition de $1 \mathrm{ml}$ d'eau.

\section{Influence de la position des feuilles traitées sur le transport des herbicides}

L'influence de la position des feuilles traitées sur le comportement du 2,4-D et du glyphosate dans le liseron a été étudiée en appliquant les herbicides radioactifs sur quatre feuilles situées dans la partie supérieure, moyenne ou inférieure des trois types de tiges des secondes repousses âgées. Les traitements ont été réalisés comme précédemment et pour chaque position, l'absorption et le transport des herbicides radioactifs ont été déterminés dans deux plantes, 11 jours après le traitement. 


\section{Statistiques}

Malgré le faible nombre de plantes étudiées (2 darıs chaque condition), les résultats ont été soumis à l'analyse de variance et les moyennes ont été comparées à l'aide du test de Duncan. Le niveau de précision étant généralement faible, la présentation des résultats se bornera souvent à dégager les tendances les plus nettes.

\section{Résultats}

Les mesures du poids de matière fraîche des fractions végétales ont permis de caractériser le développement des plantes et en particulier celui de leurs organes souterrains. Elles ont été rapportées en détail dans un document antérieur (Tamayo Esquer, 1986). Brièvement, en prenant l'exemple des plantes traitées au 2,4-D (Fig. 2), il apparaît que les radicelles constituent la majeure partie des organes souterrains des premières et secondes repousses (respectivement la moitié et les deux tiers du poids frais) et sont principalement responsabies de l'accroissement du poids frais de ces organes qui est significatif entre les deux cycles de repousse $(17,8$ vs $32,8 \mathrm{~g}$ en moyenne) mais pas entre les deux stades de développement des tiges. La masse des fractions comprises entre 0 et $40 \mathrm{~cm}$ est toujours relativement faible et ne varie pas significativement. En raison de la forme irrégulière du système souterrain des plantes, la masse de la fraction située à plus de $40 \mathrm{~cm}$ présente une grande variabilité (4 à $37 \%$ du poids frais) sans lien évident avec le stade de développement.

A tous les stades, on a pu constater que chaque herbicide avait pratiquement le même comportement dans les différents types de tige (Tamayo Esquer, 1986). En conséquence, pour chaque plante, les quantités de radioactivité mesurées à la surface des feuilles traitées des diverses tiges d'une part, dans les fractions homologues de ces tiges d'autre part, ont été additionnées de sorte que les résultats qui suivent concernent l'ensemble d'une plante.

Pour les deux herbicides, une proportion non négligeable de la radioactivité appliquée n'est pas récupérée (Tableau I). Cette proportion peut être plus élevée lorsqu'on traite les tiges âgées et s'accroître avec le temps. La majeure partie de la radioactivité appliquée reste à la surface des feuilles traitées. Dans le cas du 2,4-D, on retrouve moins de radioactivité sur les feuilles des jeunes tiges que sur celles des tiges âgées (58 vs $78 \%$

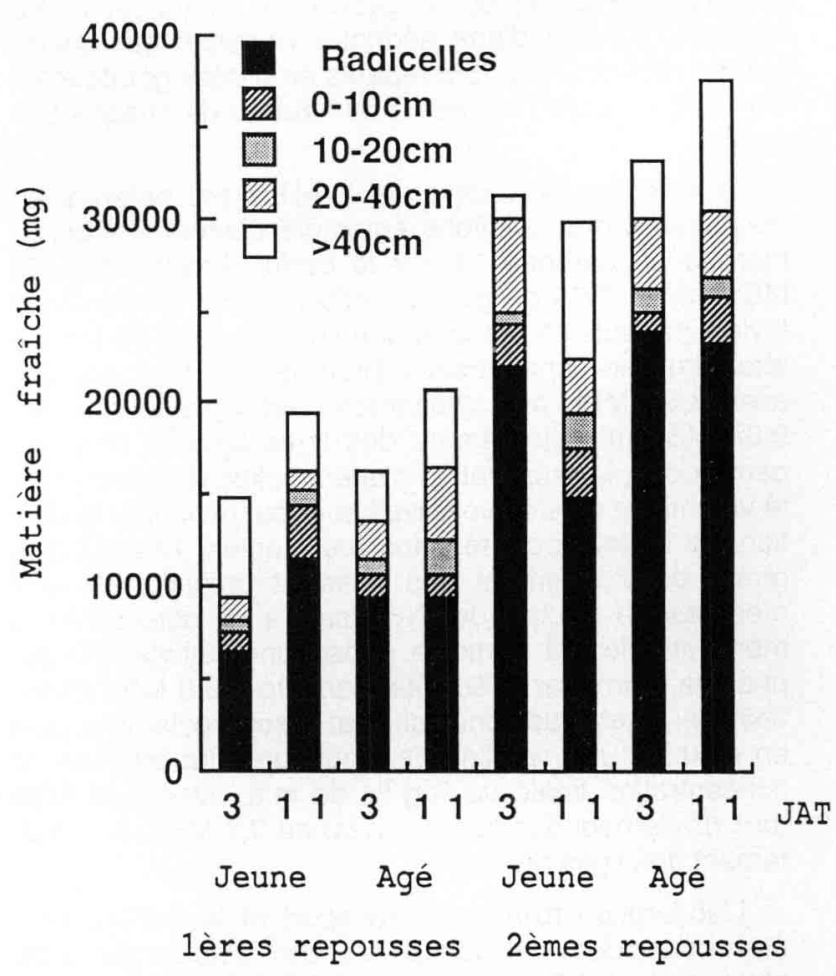

Fig. 2. Poids de matière fraîche des fractions des organes souterrains du liseron traité au 2,4-D. (JAT : jours après le traitement).

en moyenne) et les pourcentages ne diminuent, au cours du temps, que pour les jeunes premières repousses. Une faible, mais relativement constante proportion de la radioactivité appliquée est détectée dans les feuilles traitées des tiges âgées. Cette proportion est plus élevée dans les feuilles des tiges jeunes, mais elle diminue au cours du temps et rejoint celle qui est présente dans les feuilles des tiges âgées. La proportion de radioactivité appliquée qui est exportée hors des feuilles traitées est presque toujours supérieure à celle qui est présente dans les feuilles. Elle est plus grande lorsque le 2,4-D est appliqué sur les jeunes tiges que lorsqu'il est déposé sur les tiges âgées ( 23 vs $4 \%$ en moyenne) et elle augmente au cours du temps dans les jeunes premières repousses. Dans le cas du glyphosate, les proportions de radioactivité appliquée qui restent à la surface des feuilles (en moyenne 63\%), retrouvées dans les feuilles (en moyenne $7 \%$ ) et exportées (en moyenne 15\%) ne changent pas significativement en fonction des facteurs considérés. Pour cet herbicide, les mauvais bilans de radioactivité obtenus avec certaines premières repousses jeunes, trois jours après le traitement, nous ont incité à ne pas présenter les résultats correspondants. 
Tableau I. Absorption et transport du 2,4-D et du glyphosate radioactifs dans le liseron traité à différents stades de développement.

$\begin{array}{cc}\text { Feuilles traitées } & \text { Exporté Non récupéré } \\ \text { surface } & \text { intérieur } \\ \left(\% d u{ }^{14} \mathrm{C} \text { appliqué) }\right.\end{array}$

\begin{tabular}{|c|c|c|c|c|c|c|}
\hline \multicolumn{7}{|l|}{$2,4-D$} \\
\hline \multirow[t]{4}{*}{1 re rep. } & Jeune & 3JAT* & $59,5 b$ & $18,5 \mathrm{a}$ & $17,5 \mathrm{~b}$ & $4,5 b$ \\
\hline & & 11JAT & $48,5 \mathrm{c}$ & $5,5 b$ & $36,0 \mathrm{a}$ & $10,0 a b$ \\
\hline & Agé & 3JAT & $77,0 \mathrm{a}$ & $6,0 \mathrm{~b}$ & $3,0 \mathrm{c}$ & $14,0 \mathrm{a}$ \\
\hline & & 11JAT & $81,0 \mathrm{a}$ & $1,5 b$ & $2,5 \mathrm{c}$ & $15,0 \mathrm{a}$ \\
\hline \multirow[t]{4}{*}{$2^{\mathrm{e}}$ rep. } & Jeune & 3JAT & $61,5 b$ & $16,0 \mathrm{a}$ & $20,0 \mathrm{~b}$ & $2,5 \mathrm{~b}$ \\
\hline & & 11JAT & $61,5 \mathrm{~b}$ & $8,0 \mathrm{~b}$ & $18,0 \mathrm{~b}$ & $12,5 \mathrm{a}$ \\
\hline & Agé & 3JAT & $77,0 \mathrm{a}$ & $3,0 \mathrm{~b}$ & $4,0 \mathrm{c}$ & $16,0 \mathrm{a}$ \\
\hline & & 11JAT & $75,0 \mathrm{a}$ & $2,5 b$ & $6,0 \mathrm{c}$ & $16,5 \mathrm{a}$ \\
\hline \multicolumn{7}{|c|}{ Glypt osate } \\
\hline \multirow[t]{3}{*}{ 1re res. } & Jeune & 11JAT & 78,0 & 3,5 & 7,5 & $11,0 \mathrm{bc}$ \\
\hline & Agé & 3JAT & 65,5 & 7,5 & 14,5 & $12,5 b$ \\
\hline & & $11 \mathrm{JAT}$ & 51,0 & 10,0 & 16,0 & $23,0 \mathrm{a}$ \\
\hline \multirow[t]{3}{*}{$2^{e}$ rep. } & Jeune & 11JAT & 71,5 & 3,5 & 19,5 & $5,5 \mathrm{c}$ \\
\hline & Agé & 3JAT & 68,0 & 4,0 & 11,0 & $17,0 \mathrm{ab}$ \\
\hline & & 11JAT & 43,0 & 14,5 & 23,5 & $19,0 \mathrm{a}$ \\
\hline
\end{tabular}

*JAT : jours après traitement. Dans chaque colonne et pour chaque herbicide, les moyennes (2 répétitions) non suivies d'une lettre ou suivies d'une même lettre ne diffèrent pas significativement selon le test de Duncan $(P=0,05)$.

Lorsque les plantes sont traitées au 2,4-D, la radioactivité exportée gagne principalement la fraction des tiges qui porte les feuilles traitées, la partie inférieure des tiges et les organes souterrains (Tableau II). La proportion de la radioactivité exportée présente dans la première fraction tend à être plus élevée chez les jeunes tiges que chez les tiges âgées et une tendance inverse s'observe pour la seconde fraction mais peu de différences sont significatives. La radioactivité présente dans les organes souterrains se situe entre 19 et $44 \%$ de le radioactivité mobile et tend à être plus importante chez les jeunes premières repousses et les secondes repousses âgées. En général, les pourcentages tendent à diminuer au cours du temps dans la fraction des tiges qui porte les feuilles traitées et tendent à augmenter dans la partie inférieure des tiges de même que dans les organes souterrains, mais les différences sont rarement significatives. Une faible proportion de la radioactivité migre dans la partie supérieure des tiges à l'exception des jeunes secondes repousses. Enfin, une proportion négligeable de la radioactivité mobile gagne les tiges non traitées au 2,4-D radioactif.

Lorsque les plantes sont traitées au glyphosate, la majeure partie de la radioactivité exportée gagne les organes souterrains. Les proportions correspondantes, en moyenne de $62 \%$, tendent à être plus élevées chez les plantes dont les tiges sont âgées et à augmenter au cours du temps mais les différences ne sont pas significatives (Tableau II). De faibles pourcentages sont présents dans la partie des tiges qui porte les feuilles traitées ainsi que dans la partie inférieure des tiges et, pour cette dernière, ils sont toujours plus grands chez les tiges âgées. On observe que la proportion de radioactivité qui migre dans la partie supérieure des tiges est nettement plus grande chez les tiges jeunes que chez les tiges âgées ( 37,8 vs $7,1 \%$ en moyenne). Enfin, comme pour le $2,4-D$, une proportion négligeable de la radioactivité mobile gagne les tiges non traitées au glyphosate radioactif.

L'examen de la répartition de la radioactivité contenue dans les organes souterrains des plantes montre que, lorsque les tiges sont jeunes, la radioactivité correspondant au 2,4-D s'accumule principalement dans les dix premiers centimètres de ces organes (en moyenne 73\%) (Tableau III). Les radicelles renferment en moyenne $14 \%$ de la radioactivité et les autres fractions en contiennent le plus souvent très peu. Lorsque les tiges sont âgées, la radioactivité apparaît mieux distribuée 
Tableau II. Répartition de la radioactivité exportée dans le liseron traité au 2,4-D ou au glyphosate à différents stade de développement.

\begin{tabular}{lccccc}
\hline Fractions $^{*}$ & TS & $T$ & $T I$ & $T N T$ & OS \\
& & & (\% du ${ }^{14} \mathrm{C}$ exporté $)$ \\
\hline
\end{tabular}

\begin{tabular}{|c|c|c|c|c|c|c|c|}
\hline \multicolumn{8}{|l|}{ 2,4-D } \\
\hline \multirow[t]{4}{*}{$1^{\text {re rep. }}$} & Jeune & 3JAT** & $9,0 \mathrm{~b}$ & 40,5 & $18,0 \mathrm{bc}$ & 2,0 & 30,5 \\
\hline & & $11 \mathrm{JAT}$ & $6,0 \mathrm{~b}$ & 23,5 & $26,5 a b c$ & 0,0 & 44,0 \\
\hline & Agé & 3JAT & $12 a b$ & 37,0 & $31,0 \mathrm{abc}$ & 1,0 & 19,0 \\
\hline & & 11JAT & $9,0 \mathrm{~b}$ & 14,5 & $45,5 \mathrm{a}$ & 2,0 & 29,0 \\
\hline \multirow[t]{4}{*}{$2^{\mathrm{e}}$ rep. } & Jeune & 3JAT & $23,0 \mathrm{a}$ & 46,0 & $11,0 \mathrm{c}$ & 0,0 & 20,0 \\
\hline & & $11 \mathrm{JAT}$ & $20,5 \mathrm{a}$ & 40,0 & $13,5 \mathrm{bc}$ & 0,0 & 26,0 \\
\hline & Agé & 3JAT & $3,5 \mathrm{~b}$ & 24,5 & $36,5 \mathrm{ab}$ & 0,5 & 35,0 \\
\hline & & $11 \mathrm{JAT}$ & $4,0 \mathrm{~b}$ & 15,0 & $46,5 \mathrm{a}$ & 1,0 & 33,5 \\
\hline \multicolumn{8}{|l|}{ Glysphosate } \\
\hline \multirow[t]{3}{*}{ 1re rep. } & Jeune & 11JAT & $38,5 \mathrm{a}$ & 11,0 & $2,5 \mathrm{c}$ & 3,0 & 45,0 \\
\hline & Agé & 3JAT & $4,5 \mathrm{~b}$ & 14,0 & 24,5 a & 1,0 & 56,0 \\
\hline & & 11JAT & $9,0 \mathrm{~b}$ & 9,0 & $12,5 b$ & 0,5 & 69,0 \\
\hline \multirow[t]{3}{*}{$2^{\mathrm{e}}$ rep. } & Jeune & $11 \mathrm{JAT}$ & $37,0 \mathrm{a}$ & 4,5 & $1,0 \mathrm{C}$ & 0,5 & 57,0 \\
\hline & Agé & 3JAT & $11,0 \mathrm{~b}$ & 8,0 & $13,5 \mathrm{~b}$ & 1,5 & 66,0 \\
\hline & & 11JAT & $4,0 \mathrm{~b}$ & 5,5 & $10,0 \mathrm{~b}$ & 0,5 & 80,0 \\
\hline
\end{tabular}

Tableau III. Répartition de la radioactivité dans les organes souterrains du liseron traité au 2,4-D ou au glyphosate à différents stades de développement.

\begin{tabular}{|c|c|c|c|c|c|}
\hline \multirow{2}{*}{ Fractions ${ }^{*}$} & $R$ & $0-10$ & $10-20$ & $20-40$ & $>40$ \\
\hline & & \multicolumn{4}{|c|}{ (\% du ${ }^{14} \mathrm{C}$ des organes souterrains) } \\
\hline
\end{tabular}

\begin{tabular}{|c|c|c|c|c|c|c|c|}
\hline \multicolumn{8}{|l|}{ 2,4-D } \\
\hline \multirow[t]{4}{*}{ 1re rep. } & Jeune & $3 \mathrm{JAT}^{\star *}$ & $11,5 \mathrm{~b}$ & $83,5 a$ & $2,0 \mathrm{bc}$ & $2,0 \mathrm{c}$ & 1,0 \\
\hline & & 11JAT & $15,5 \mathrm{~b}$ & $58,0 \mathrm{~b}$ & $12,5 \mathrm{~b}$ & $12 a b c$ & 2,0 \\
\hline & Agé & 3JAT & $34,0 \mathrm{ab}$ & $25,0 \mathrm{C}$ & $7,0 \mathrm{~b}$ & $21,5 a b$ & 12,5 \\
\hline & & 11JAT & $17,5 \mathrm{ab}$ & $24,0 \mathrm{C}$ & $25,0 \mathrm{a}$ & $16,5 a b c$ & 17,0 \\
\hline \multirow[t]{4}{*}{$2^{e}$ rep. } & Jeune & 3JAT & $18,5 \mathrm{ab}$ & $71,5 \mathrm{ab}$ & $3,5 \mathrm{~b}$ & $6,5 \mathrm{c}$ & 0,0 \\
\hline & & 11JAT & $11,5 \mathrm{~b}$ & $80,5 a$ & $3,5 \mathrm{~b}$ & $2,0 \mathrm{C}$ & 2,5 \\
\hline & Agé & 3JAT & $38,5 \mathrm{a}$ & $19,5 \mathrm{c}$ & $11,0 \mathrm{~b}$ & $24,0 \mathrm{a}$ & 7,0 \\
\hline & & 11JAT & $23,5 a b$ & $34,5 \mathrm{c}$ & $14,0 a b$ & $19,0 \mathrm{ab}$ & 9,0 \\
\hline \multicolumn{8}{|l|}{ Glyphosate } \\
\hline \multirow[t]{3}{*}{1 re rep. } & Jeune & 11JAT & 64,0 & 11,0 & $10,5 \mathrm{a}$ & 4,5 & 10,0 \\
\hline & Agé & 3JAT & 77,0 & 9,5 & $7,0 a b c$ & 5,5 & 1,0 \\
\hline & & 11JAT & 45,0 & 3,5 & $4,0 \mathrm{c}$ & 7,5 & 40,0 \\
\hline \multirow[t]{3}{*}{$2^{\theta}$ rep. } & Jeune & $11 \mathrm{JAT}$ & 56,5 & 8,0 & $9,0 a b$ & 9,5 & 17,0 \\
\hline & Agé & 3JAT & 44,0 & 7,0 & $5,5 \mathrm{bc}$ & 8,5 & 35,0 \\
\hline & & $11 \mathrm{JAT}$ & 57,0 & 5,5 & $5,0 \mathrm{c}$ & 11,0 & 23,0 \\
\hline
\end{tabular}

" $R$ : radicelles; éloignement de la base des tiges $(\mathrm{cm})$.

"*JAT : jours après le traitement.

Dans chaque colonne et pour chaque herbicide, les moyennes (2 répétitions) non suivies d'une lettre ou suivies d'une même lettre ne diffèrent pas significativement selon le test de Duncan $(P=0,05)$. 
dans l'ensemble des fractions considérées. Dans le cas du glyphosate, une forte proportion de la radioactivité ( $57 \%$ en moyenne) est toujours présente dans les radicelles. Les fractions situées entre 0 et $40 \mathrm{~cm}$ contiennent chacune environ $7 \%$ de la radioactivité. Les pourcentages retrouvés dans la fraction située au-delà de $40 \mathrm{~cm}$ varient considérablement ( 1 à $40 \%$ ) selon la taille de cette fraction.

Les procédures d'extraction ont permis de récupérer plus de $95 \%$ de la radioactivité contenue dans les fractions analysées des plantes traitées au 2,4-D ou au glyphosate. Malgré l'absence de répétition, il est possible de formuler les remarques suivantes. Le métabolisme du 2,4-D ne semble dépendre ni du stade de développement des plantes ni de la fraction végétale considérées (Tamayo Esquer, 1986). On peut indiquer (données non présentées) que l'herbicide est bien dégradé puisque moins de $40 \%$ de la radjoactivité extraite correspondent au composé initial. On retrouve principalement des métabolites solubles dans l'éther dont le plus important est immobile dans le premier système chromatographique tandis que trois autres composés, de faible $R_{f}$ et détectés à l'état de traces, pourraient être des conjugués aminoacides du 2,4-D. Les métabolites hydrosolubles ne représéntent en moyenne que $22 \%$ de la radioactivité extractible et sont immobiles dans le premier système chromatographique. L'hydrolyse acide des métabolites solubles dans l'éther ou dans l'eau est toujours complète et libère un seul composé soluble dans l'éther dont le $R_{f}$ correspond à celui de 2,4-D. Le métabolisme du glyphosate dans les plantes n'a jamais pu être mis en évidence.
L'application du 2,4-D radioactif sur des feuilles situées à différents niveaux des tiges âgées des secondes repousses révèle que seul le dépôt de l'herbicide sur les feuilles supérieures permet un transport acropète important de la radioactivité (Tableau IV). D'une manière générale, le transport est principalement basipète et la proportion de radioactivité mobile qui gagne les organes souterrains augmente nettement lorsque la distance entre les feuilles traitées et ces organes diminue. Quelle que soit la position des feuilles traitées, le transport de la radioactivité correspondant au glyphosate est toujours fortement basipète. Plus de $69 \%$ de la radioactivité mobile atteint les organes souterrains, et cette proportion tend à s'accroître lorsque les feuilles sont plus proches de la base des tiges.

\section{Discussion}

Dans cette étude, les plantes présentent la particularité de posséder un très grand nombre de radicelles qui constituent une fraction importante des organes souterrains. L'évolution du poids frais des fractions de ces organes suggère que la croissance, principalement assurée par les radicelles, se produit surtout avant le traitement des jeunes tiges et sans doute au début de chaque cycle de repousse.

Le taux de récupération de la radioactivité est médiocre mais pourtant comparable à celui obtenu dans des études similaires (Coble et al., 1970). Dans le cas du 2,4-D, les pertes peuvent se produire par volatilisation à la surface des feuilles et surtout par exsudation racinaire comme on a pu

Tableau IV. Influence de la position des feuilles traitées sur la répartition de la radioactivité exportée dans les secondes repousses âgées du liseron traité au 2,4-D ou au glyphosate.

\begin{tabular}{|c|c|c|c|c|c|}
\hline Fractions ${ }^{*}$ & TS & $T$ & $\begin{array}{l}\text { TI } \\
\text { C expor }\end{array}$ & TNT & os \\
\hline 2,4-D & & & & & \\
\hline $\begin{array}{c}\text { Position haute } \\
\text { moyenne } \\
\text { basse }\end{array}$ & $\begin{array}{r}19,5 \mathrm{a} \\
4,0 \mathrm{~b} \\
5,5 \mathrm{~b}\end{array}$ & $\begin{array}{l}27,9 \mathrm{ab} \\
15,0 \mathrm{bc} \\
43,0 \mathrm{a}\end{array}$ & $\begin{array}{l}37,5 \mathrm{a} \\
46,5 \mathrm{a} \\
-\end{array}$ & $\begin{array}{l}1,5 \mathrm{~b} \\
1,0 \mathrm{~b} \\
5,5 \mathrm{a}\end{array}$ & $\begin{array}{l}13,6 \mathrm{~b} \\
33,5 \mathrm{ab} \\
46,0 \mathrm{a}\end{array}$ \\
\hline Glyphosate & & & & & \\
\hline $\begin{array}{l}\text { Position haute } \\
\text { moyenne }\end{array}$ & $\begin{array}{l}8,5 \\
4,0\end{array}$ & $\begin{array}{l}7,5 \\
5,5\end{array}$ & $\begin{array}{l}14,0 \\
10,0\end{array}$ & $\begin{array}{l}1,0 \\
0,5\end{array}$ & $\begin{array}{l}69,0 \\
80,0\end{array}$ \\
\hline
\end{tabular}

"Voir Tableau II. Dans chaque colonne et pour chaque herbicide, les moyennes (2 répétitions) non suivies d'une lettre ou suivies d'une même lettre ne differrent pas significativement selon le test de Duncan $(P=0,05)$. 
l'observer chez Cirsium arvense L. (Turnbull et Stephenson, 1985) et Ampelamus albidus Nutt. Britt. (Coble et al., 1970). Des essais préliminaires tendent à écarter l'hypothèse d'une volatilisation du glyphosate hydrosoluble qui pourrait être perclu pendant le lavage des organes souterrains, au moment de la récolte, mais aussi par exsudation racinaire comme le suggèrent les expériences de Marquis et al. (1979).

Dans la plupart des conditions, l'absorption, le transport et le métabolisme des herbicides sont relativement rapides et se produisent pendant les trois premiers jours qui suivent le traitement. Dans le cas du 2,4-D, l'examen des quantités de radioactivité présentes à la surface des feuilles et retrouvées dans les plantes, suggère que l'absorption de cet herbicide est plus grande dans les feuilles des tiges jeunes que dans celles des tiges âgées. Ce résultat est conforme aux observations antérieures montrant que l'absorption foliaire des herbicides peut diminuer au cours du développement des feuilles et des plantes (Sargent et Blackman, 1972; Ahmadi et al., 1980; Duncan et al., 1981; Baker et Hunt, 1981; Soteres et al., 1983). II pourrait être dû à une baisse de la perméabilité cuticulaire qui accompagne souvent le développement des feuilles. En conséquence, de moins grandes quantités de radioactivité sont exportées et s'accumulent dans les fractions des plantes traitées à la floraison. Contrairement au 2,4-D, l'absorption foliaire du glyphosate ne change pas avec le stade de développement des tiges. Ce résultat, plutôt surprenant, pourrait s'expliquer par la forte humidité relative de la chambre climatisée $(85 \%)$, qui favorise particulièrement la pénétration de cet herbicide (Gottrup et al., 1976; Wills, 1978).

Pour les deux herbicides, la radioactivité qui pénètre dans les plantes est bien exportée hors des feuilles traitées. Le transport est principalement basipète et le glyphosate atteint mieux les organes souterrains que le 2,4-D. II dépend cependant du stade de développement des tiges mais aussi, dans le cas du 2,4-D, du cycle de repousse. Lorsque cet herbicide est appliqué sur de jeunes tiges, un transport acropète significatif ne se produit que dans les secondes repousses, et la radioactivité qui migre dans les organes souterrains s'accumule surtout dans la partie superficielle (moins de $10 \mathrm{~cm}$ ). Lorsque l'herbicide est appliquée sur des tiges âgées, le transport est presque exclusivement basipète et la radioactivité est mieux distribuée dans les organes souterrains. A ce stade, seules les feuilles situées à proximité de l'apex des tiges permettent un transport acropète de la radioactivité. Le transport du glyphosa- te, quant à lui, ne dépend que du stade de développement des tiges. Il présente une composante acropète dans les jeunes tiges alors qu'il est presque exclusivement basipète dans les tiges âgées, sans incidence notable de la position des feuilles traitées.

Ces résultats sont comparables à ceux d'autres auteurs qui ont observé un accroissement du transport basipète des herbicides hormonaux, dans les tiges âgées de Convolvulus arvensis $L$. (Muller et Guncan, 1973) et du clopyralide dans celles de Cirsium arvense L. $\left(O^{\prime}\right.$ Sullivan et Kossatz, 1984) ainsi que l'accumulation du glyphosate dans les organes souterrains de Cynodon dactylon L. (Whitwell et al., 1980), Agropyrum repens $L$. (Devine et al., 1983) et Cirsium arvense L. (McAllister et Haderlie, 1985). Ils sont compatibles avec un transport des herbicides dans le phloème, permettant des mouvements acropètes, vers l'extrémité des tiges - qui se réduisent - et surtout basipètes, vers les organes souterrains, qui s'accroissent lorsque les tiges se développent. Cependant, si le transport paraît lié au développement des tiges, on peut penser que le rôle des organes souterrains du liseron est déterminant. Sur la base d'un mécanisme de transport de type "source-puits", ces organes peuvent constituer un puits très important dont l'activité est susceptible d'augmenter avec la croissance et la constitution de réserves, et qui pourrait être par conséquent responsable des phénomènes observés. Le faible transport acropète du 2,4-D, observé dans les jeunes premières repousses, pourrait alors être lié à la croissance des organes souterrains encore peu développés mais peut-être capables de drainer plus fortement les assimilats que ceux, plus importants, des secondes repousses. Ainsi, la croissance des organes souterrains du liseron pourrait influencer le transport du 2,4-D, comme l'ont observé Coble et al. (1970) chez Ampelamus albidus, plus spécialement lorsque leur taille est réduite. Le transport du glyphosate semble moins dépendant de ce phénomène. Pour cet herbicide, les quantités importantes de radioactivité retrouvées dans la partie supérieure des jeunes tiges et dans les radicelles confortent l'hypothèse de son attraction par les zones de croissance (Sprankle et al., 1975; Sandbert et al., 1980; Whitwell et al., 1980; Devine et al., 1983). Toutefois, son accumulation relativement uniforme (lorsqu'on tient compte de la masse des fractions) dans l'ensemble des organes souterrains constitue un autre fait remarquable. Elle suggère que cet herbicide est également capable de s'accumuler dans les organes de réserve. 
Le 2,4-D est rapidement dégradé dans le liseron et transformé principalement en métabolites solubles dans l'éther. Le plus important de ces métabolites a un comportement chromatographique analogue à celui d'un dérivé non identifié du MCPA, soluble dans l'éther et trouvé dans plusieurs dicotylédones (Cole et Loughman, 1983). Les résultats de l'hydrolyse suggèrent qu'il peut s'agir d'un complexe formé à partir du 2,4-D. Comme les conjugués aminoacides, il est probablement constitué de molécules ioniques puisqu'un $\mathrm{pH}$ bas est nécessaire à l'efficacité du partage éther/eau, mais la nature des molécules reste à préciser. L'hydrolyse suggère également que les métabolites hydrosolubles pourraient être des conjugués de type glucose-ester du 2,4-D de sorte que le liseron apparaît, comme beaucoup d'autres dicotylédones, incapable d'hydroxyler les molécules du 2,4-D ce que font au contraire les graminées (Bristol et al., 1977). La vitesse et le taux de métabolisme du 2,4-D dans le liseron sont susceptibles de limiter le transport et par conséquent l'action de cet herbicide dans les plantes. A l'opposé, la stabilité du glyphosate est favorable au transport et à l'activité de ce dernier.

D'un point de vue pratique, les résultats confirment le meilleur transport basipète du 2,4-D et du glyphosate dans le liseron lorsque ces herbicides sont appliqués sur des tiges âgées. De plus, à ce stade, un plus grand nombre de feuilles peuvent exporter les herbicides vers les organes souterrains des plantes ce qui devrait contribuer à renforcer l'efficacité des traitements même si les quantités de matières actives qui atteignent ces organes diminuent lorsque la distance à parcourir augmente. Toutefois, une faible absorption foliaire, observée notamment dans le cas du 2,4-D, peut réduire considérablement les avantages de transport qu'offrent les traitements tardifs, en diminuant la quantité de matière active présente dans les plantes et en défavorisant par conséquent son accumulation dans les organes souterrains.

\section{Références}

Aberg E. \& Stecko V. (1976) Internal factors affecting toxicity. In : Herbicide Physiology Biochemistry Ecology (Audus, ed.), Academic Press, London, pp. 24-28

Agbakoba C.S.O. \& Goodin J.R. (1969) Effect of stage of growth of field bindweed on absorption and translocation of ${ }^{14} \mathrm{C}$-labelled 2,4-D and picloram. Weed Sci. 17, 436-438

Ahmadi M.S., Haderlie L.C. \& Wicks G.A. (1980) Effect of growth stage and water stress on barnyardgrass
(Echinochloa crusgalli) control and on glyphosate absorption and translocation. Weed Sci. 28, 277-282

Baker E.A. \& Hunt G.M. (1981) Developmental changes in leaf epicuticular waxes in relation to foliar penetration. New Phytol. 88, 731-747

Bristol D.W., Ghanuni A.M. \& Oleson A.E. (1977) Metabolism of 2,4-dichlorophenoxyacetic acid by wheat cell suspension cultures. J. Agric. Food Chem. 25, 1308-1313

Coble H.D., Slife F.W. \& Butler H.S. (1970) Absorption, translocation and metabolism of 2,4-D by honeyvine milkweed. Weed Sci. 18, 653-656

Cole D.J. \& Loughman B.C. (1983) The metabolic fate of (4-chloro-2-methyl) acetic acid in higher plants. $J$. Exp. Bot. 34, 1299-1310

Coupland D. (1984) The effect of temperature on the activity and metabolism of glyphosate applied to rhizome fragments of Elymus repens (=Agropyrum repens). Pestic. Sci. 15, 226-234

Devine M.D., Bandeen J.D. \& McKersie B.D. (1983) Temperature effects on glyphosate absorption, translocation and distribution in quackgrass (Agropyrum repens). Weed Sci. 31, 461-464

Duncan D.N., Meggitt F.W. \& Penner D. (1981) Physiological bases of sugar beet (Beta vulgaris) tolerance to foliar application of ethofumesate. Weed Sci. 29, 648-654

Gottrup O., O'Sullivan P.A., Schraa R.J. \& Van Den Born W.H. (1976) Uptake, translocation, metabolism and selectivity of glyphosate in Canada thistle and leafy spurge. Weed Res. 16, 197-201

Hamilton R.H., Hurter J., Hall J.K. \& Ercegovich C.D. (1971) Metabolism of 2,4-dichlorophenoxyacetic acid and 2,4,5-trichlorophenoxyacetic acid by bean plants. J. Agric. Food Chem. 19, 480-483

Legay J.J. \& Tyburn C. (1981) Mode of action of an auxin antagonist : the 2,4,6-trichlorophenoxyacetic acid inhibitor of the growth of Acer pseudoplatanus L. cell suspensions. Physiol. Vég. 19, 491-502

Lolas P.C. \& Coble H.D. (1980) Translocation of ${ }^{14} \mathrm{C}-$ glyphosate in johnsongrass (Sorghum halepense L. Pers.) as affected by growth stage and rhizome length. Weed Res. 20, 267-270

Marquis L.Y., Comes R.D. \& Yang C.P. (1979) Selectivity of glyphosate in creeping red fescue and red canarygrass. Weed Res. 19, 335-342

McAllister R.S. \& Haderlie L.C. (1985) Translocation of ${ }^{14} \mathrm{C}$-glyphosate and ${ }^{14} \mathrm{CO}_{2}$-labeled photoassimilates in Canada thistle (Cirsium arvense). Weed Sci. 33, 153159

Muller F. \& Guncan A. (1973) Translocation of ${ }^{14}$ C-marked herbicides in Convolvulus arvensis $\mathrm{L}$. at various growth stages. Deutsche Pflanzenschutz-Taung der biologischen Bundesanstalt fur Land und Forstwirstchaft, Stuttgart, 1973). Mitt. Biol. Bundesanst. Land Forstwirtsch. Berl. Dahlem 151, 191-192

O' Sullivan P.A. \& Kossatz V.C. (1984) Absorption and translocation of ${ }^{14} \mathrm{C}-3,6$-dichloropicolinic acid in Cirsium arvense L. Scop. Weed Res. 24, 17-22

Sandberg C.L., Meggitt W.F. \& Penner D. (1980) Absorption, translocation and metabolism of ${ }^{14} \mathrm{C}$-glyphosate in several weed species. Weed Res. 20, 195-200 
Sargent J.A. \& Blackman G.E. (1972) Studies on foliar penetration. IX. Patterns of penetration of 2,4-dichlorophenoxyacetic acid into leaves of different species. J. Exp. Bot. 23, 830-841

Soteres J.K., Murray D.S. \& Basler E. (1983) Absorption of 2,4-D, dicamba and glyphosate by excised honeywine milkweed (Cinanchum laeve) leaves. Weed Sci. 31, 271-274

Sprankle P., Meggitt W.F. \& Penner D. (1975) Absorption, accumulation and translocation of glyphosate. Weed Sci. 23, 235-240

Sprankle P., Sandberg C.L., Meggitt W.F. \& Penner D. (1978) Separation of glyphosate and possible metabolites by thin layer chromatography. Weed Sci. 26, 673-674

Tamayo Esquer L.M. (1986) Etude de la dynamique de population de Convolvulus arvensis L. Action et comportement du 2,4-D et du glyphosate dans la plante. Thèse de Docteur Ingénieur. Ecole Nationale Supérieure Agronomique de Montpellier

Turnbull G.C. \& Stephenson G.R. (1985) Translocation of clopyralid and 2,4-D in Canada thistle (Cirsium arvense). Weed Sci. 33, 143-147

Whitwell T., Banks P., Basler E. \& Santerlman P.W. (1980) Glyphosate absorption and translocation in bermudagrass (Cynodon dactylon) and activity in hornsettle (Solanum carolinensise). Weed Sci. 28, 93-96

Wills G.D. (1978) Factors affecting toxicity and translocation of glyphosate in cotton (Gossypium hirsutum). Weed Sci. $26,509-513$ 